\title{
Heck-oxyarylation of 2-phenyl-2H-chromenes and 1,2-dihydronaphthalenes
}

\author{
Katalin Gulácsi, ${ }^{a}$ István Németh, ${ }^{a}$ Ádám Szappanos, ${ }^{a}$ Kinga Csillag, ${ }^{a}$ \\ Tünde Zita Illyés, ${ }^{a}$ Tibor Kurtán, ${ }^{a}$ and Sándor Antus ${ }^{\mathrm{a}, \mathrm{b}, *}$ \\ ${ }^{a}$ Department of Organic Chemistry, University of Debrecen, H-4010 Hungary, Debrecen, P.O. Box 20 \\ ${ }^{\mathrm{b}}$ Carbohydrate Research Group of Hungarian Academy Sciences, H-4010 Hungary, Debrecen, P.O. Box 20
}

RECEIVED MAY 29, 2012; REVISED OCTOBER 10, 2012; ACCEPTED OCTOBER 15, 2012

\begin{abstract}
The Heck-oxyarylation of racemic 2-phenyl-2H-chromene $[( \pm)-\mathbf{4 b}]$ and 1,2-dihydronaphthalenes $(\mathbf{1 4 a}, \mathbf{b})$ has been studied with 2-chloromercuriphenols $(\mathbf{5 a}-\mathbf{d})$ in the presence of $\mathrm{Li}_{2}\left[\mathrm{PdCl}_{4}\right]$ catalyst. The reactions resulted in the diastereoselective formation of racemic 6phenylpterocarpans of $(6 R, 6 \mathrm{a} R, 11 \mathrm{a} R)$ relative configuration $[( \pm)-8 \mathbf{a}-\mathbf{d}]$ and their dibenzo[1,3]dioxocine analogues $[( \pm)-\mathbf{1 2 a}-\mathbf{d}]$ as main products, respectively. The ratio of products and the lack of regioisomeric products (13a-d) corroborated the cationic mechanism of the oxyarylation of $2 \mathrm{H}$-chromenes, which has been also supported by the transformation of 14a,b under similar conditions.(doi: 10.5562/cca2103)
\end{abstract}

Keywords: Pterocarpans; Dibenzo[d,g][1,3]dioxocines; Palladium(0); Oxidative coupling; Reaction mechanism; Catalysis

\section{INTRODUCTION}

Pterocarpans are naturally occurring plant products containing a cis-fused benzofurano-benzopyran skeleton. Many of them are phytoalexins produced in plants during infections by fungi, viruses or bacteria and subsequently act as protective agents for plants. ${ }^{1}$ Moreover, some representatives of this type of natural products have significant oestrogenic activity ${ }^{2}$ and others have been reported to inhibit HIV-1 reverse transcriptase in cell cultures ${ }^{3}$ and to possess high activity against snake or spider venoms. ${ }^{4}$

Among the wide variety of synthetic routes to racemic pterocarpans $[( \pm)-3],{ }^{5-16}$ one of the most commonly used approach ${ }^{17-22}$ is based on the Heckoxyarylation of $2 \mathrm{H}$-chromenes (1) with 2-chloromercuriphenols (2) using equimolar amount of $\mathrm{Li}_{2}\left[\mathrm{PdCl}_{4}\right]$ as catalyst (Scheme 1). ${ }^{7}$
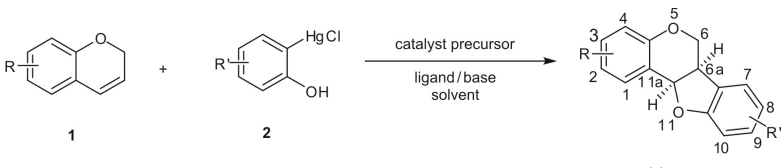

$( \pm)-3$
In contrast to the reports of Breytenbach ${ }^{17}$ we have found that the oxyarylation of 7-benzyloxy- $2 \mathrm{H}$ chromene (4a) with 2-chloromercuri-3,4-methylenedioxyphenol (5a) under the conditions published by Horino and Inoue ${ }^{7}$ did not take place with complete regioselectivity $(\mathbf{4} \mathbf{a}+5 \mathbf{a} \rightarrow \mathbf{6 a} \rightarrow \mathbf{7 a} \rightarrow \mathbf{8 a})$, but additional coupled products 12a and 13a were also obtained (Scheme 2), probably via $10 a \rightarrow 11 a$ and 10a carbocation intermediates, respectively. ${ }^{23}$

In order to study the factors that determine the nature and ration of the three possible products, we studied the effect of an additional $\mathrm{C}-2$ phenyl group on the $2 \mathrm{H}$ chromene ring and replacement of its oxygen by a methylene group as well as different substitution pattern of the 2-chloromercuriphenol. Thus Heck-oxyarylations of racemic 2-phenyl-2H-chromene $[( \pm)-4 \mathbf{b}]$ and 1,2dihydronaphthalenes $(\mathbf{1 4 a}, \mathbf{b})$ were carried out with three 2-chloromercuriphenols $(\mathbf{5 a}-\mathbf{c})$ in the presence of $\mathrm{Li}_{2}\left[\mathrm{PdCl}_{4}\right]$ at room temperature.

\section{RESULTS AND DISCUSSION}

The racemic 2-phenyl-2H-chromene $[( \pm)-\mathbf{4 b}]$ was obtained from rac-flavanone in two steps according to literature. ${ }^{24}$ 2-Chloromercuriphenols $(\mathbf{5 a}-\mathbf{d})$ were prepared from commercially available sesamol $(3,4-$

Scheme 1. Heck-oxyarylation of $2 H$-chromenes.

\footnotetext{
* Author to whom correspondence should be addressed. (E-mail: antus.sandor@science.unideb.hu)
} 


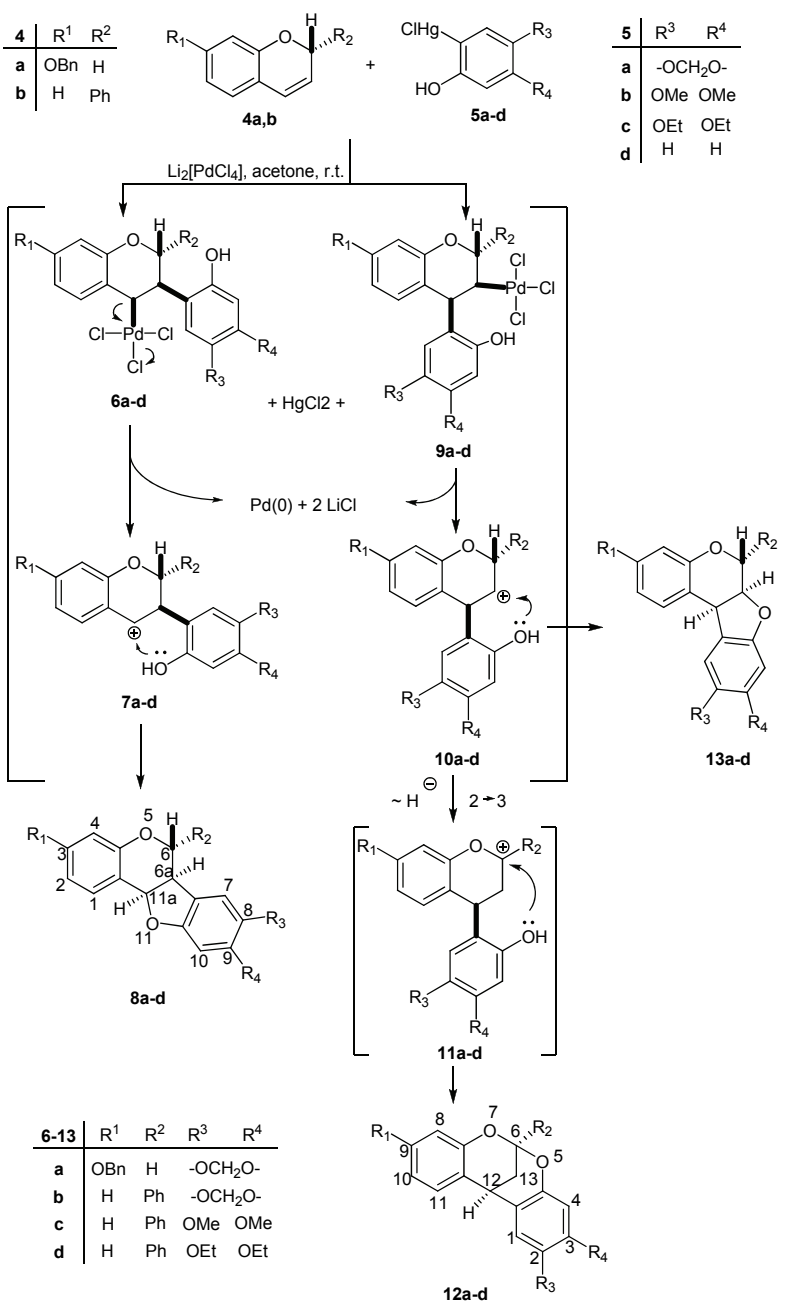

Scheme 2. Proposed sequence of $\operatorname{Pd}(0)$-catalyzed oxyarylation of $\mathbf{4 a}, \mathbf{b}$ with $\mathbf{5 a - c .}$

methylendioxyphenol), 3,4-dimethoxyphenol and 3,4diethoxyphenol and phenol by mercury (II) acetate, respectively, under the conditions used earlier by two of us. $^{23}$ The TLC monitoring of oxyarylation of $( \pm)-\mathbf{4 b}$ with 5a has clearly shown that the conversion of the starting material $[( \pm)-\mathbf{4 b}]$ reached about $50 \%$ in three hours and then changed very slowly with longer reaction time.

Besides the starting material $( \pm)-\mathbf{4 b}$, two main products could be isolated by column chromatography whose structures were elucidated by spectroscopic methods. On the basis of ${ }^{1} \mathrm{H},{ }^{13} \mathrm{C}$ NMR and MS data, the major product could be identified as racemic 6phenyl-8,9-methylenedioxypterocarpan $[( \pm)-8 \mathbf{b}]$. The large coupling constant between of H-6 and H-6a ( $\mathrm{J}=$ $10.8 \mathrm{~Hz}$ ) has clearly indicated the trans diaxial orientation of H-6, H-6a and thus the trans relative configuration of the C- 6 phenyl and C-6a aryl groups. The addition of the organopalladium intermediate formed from
Table 1. Compounds formed by oxyarylation of olefin $4 \mathbf{a}, \mathbf{b}$

\begin{tabular}{lccc}
\hline $\begin{array}{l}\text { Entry } \\
\text { Olefin }+\end{array}$ & $\begin{array}{c}\text { Product } \\
(\text { Yield } / \%)\end{array}$ & $\begin{array}{c}\text { Ratio of } \\
\text { product }\end{array}$ \\
\hline 1 & $\mathbf{4 a}+\mathbf{5 a}$ & $\mathbf{8 a}(53) ; \mathbf{1 3 a}(3) ; \mathbf{1 2 a}(7.5)$ & $\mathbf{8 a}: \mathbf{1 2 a}=7: 1$ \\
2 & $\mathbf{4 b}+\mathbf{5 a}$ & $\mathbf{8 b}(8) ; \mathbf{1 3 b}($ n.d. $) ; \mathbf{1 2 b}(2)$ & $\mathbf{8 b}: \mathbf{1 2 b}=4: 1$ \\
3 & $\mathbf{4 b}+\mathbf{5 b}$ & $\mathbf{8 c}(13) ; \mathbf{1 3 c}($ n.d.); $\mathbf{1 2 c}(5)$ & $\mathbf{8 c}: \mathbf{1 2 c}=2.4: 1$ \\
4 & $\mathbf{4 b}+\mathbf{5 c}$ & $\mathbf{8 d}(20) ; \mathbf{1 3 d}($ n.d. $) ; \mathbf{1 2 d}(23)$ & $\mathbf{8 d}: \mathbf{1 2 d}=1: 1.1$ \\
\hline (a) & isolated yields; n.d. - not detected. &
\end{tabular}

5a by $\mathrm{Li}_{2}\left[\mathrm{PdCl}_{4}\right]$ took place at C-3 of $( \pm)-\mathbf{4 b}$ diastereoselectively, from the opposite side to the $\mathrm{C}-2$ phenyl group to give racemic 6-phenylpterocarpan $[( \pm)-8 \mathbf{b}]$ with $(6 S, 6 \mathrm{a} R, 11 \mathrm{a} R)$ relative configuration. The other product was identified as $( \pm)-6$-phenyl-6,11-methano-2,3-methylendioxy- $6 H$-dibenzo[d,g][1,3]dioxocine $[( \pm)-\mathbf{1 2 b}]$ by comparing its NMR and MS data with those of $[( \pm)-\mathbf{1 2 a}] .^{23}$ Due to its bridged structure, the C-6 phenyl and H-12 adopt necessarily cis equatorial orientations implying a $(6 R, 12 R)$ relative configuration. It is to be noted that neither the diastereomer of $( \pm)-8 \mathrm{~b}$ with $(6 R, 6 \mathrm{a} R, 11 \mathrm{a} R)$ relative configuration, nor the regioisomeric $( \pm)$-13b bearing the 3,4-methylenedioxyphenyl group at $\mathrm{C}-4$ of the flavane skeleton could be isolated.

Comparison of the recent findings with our previous observations ${ }^{23}$ allowed following conclusions: (i) the Heck-type oxyarylation of $( \pm)-4 b$ did not take place with complete regioselectivity leading to the formation of both palladium intermediates $\mathbf{6 b}$ and $9 \mathbf{b}$, (ii) the ring-closure of the latter via the corresponding carbocationic intermediates $\mathbf{1 0 b}$ and $\mathbf{1 1 b}$ resulted in the bridged product $( \pm)-\mathbf{1 2 b}$. Since the formation of the $( \pm)$-13b regioisomer of $( \pm)-\mathbf{8 b}$ could not be observed, one may assume that the life time of the cationic intermediate 10b must be very short due to its rapid transformation by a 1,2 hydride-shift to $\mathbf{1 1 b}$. The formation of the tertiary carbocation $\mathbf{1 1 b}$ is clearly enhanced by the presence of the C-2 phenyl group. Accordingly, the ratio of the products (8b:12b) (Table 1. entry 2) is significantly smaller than that of (8a:12a) (entry 1) obtained in the reaction of 7-benzyloxy- $2 \mathrm{H}$-chromene (4a) with $5 \mathbf{a}^{23}$

The formation of the bridged product was also facilitated further by using the $\mathbf{5 b}$ or $\mathbf{5 c}$ dialkoxy chloromercuriphenols possessing an increased nucleophilicty of their hydroxy group (entry 3 and 4). Thus the proposed cationic mechanism of the Heckoxyarylation of $2 \mathrm{H}$-chromenes (1) with 2-chloromercuriphenols (2) in the presence of of $\mathrm{Li}_{2}\left[\mathrm{PdCl}_{4}\right]$ catalyst is justified.

The role of the chromene oxygen in the Heckoxyarylation was studied in the reaction of 1,2dihydronaphthalenes $(\mathbf{1 4 a}, \mathbf{b})$ with 2-chloromercuriphenols $\mathbf{( 5 a , d )}$ under the conditions discussed above (Scheme 3). 


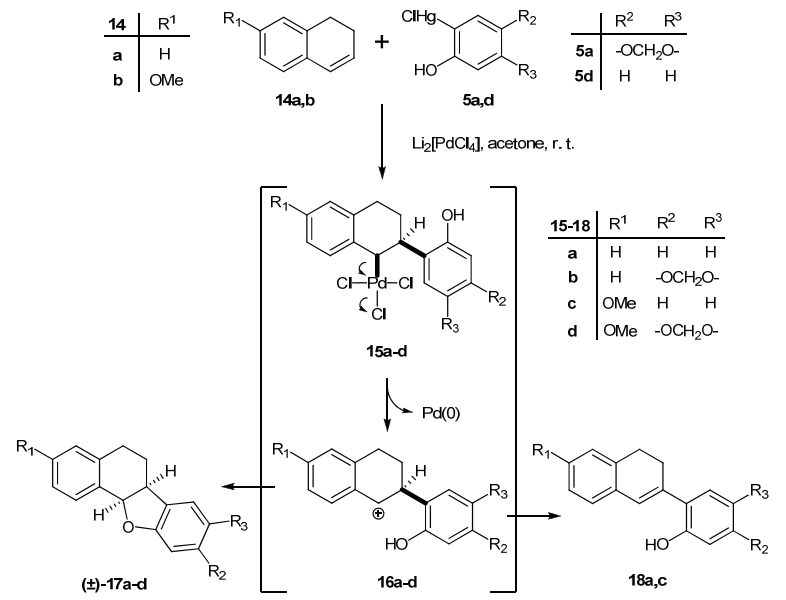

Scheme 3. Proposed sequence of $\operatorname{Pd}(0)$-catalyzed oxyarylation of 14a,b with 2 and $5 \mathbf{a}$.

In the reaction of 1,2-dihydronaphthalene (14a) and 2-chloromercuriphenol (5d), the formation of 5carbapterocarpan $[( \pm)-\mathbf{1 7} \mathbf{a}]$ and traces of 3-(2hydroxyphenyl)-1,2-dihydronaphthalene (18a) were observed. When an electron rich 2-chloromercuriphenol derivative, such as $\mathbf{5 a}$ was used instead of $\mathbf{5 d}$, the yield of the heteroannulation product $( \pm)-\mathbf{1 7} \mathbf{b}$ improved and the corresponding side-product $\mathbf{1 8 b}$ could not be detected. The transformation of the electron rich dihydronaphthalene (14b) with 5a took place in a similar manner to result in $\mathbf{1 7 d}$. On the other hand, its oxyarylation with $\mathbf{5 d}$ resulted in $( \pm)-\mathbf{1 7} \mathbf{c}$ as the minor product and the so called Heck-type product (18c) was isolated in $48 \%$ yield. Thus the syn-arylpalladation of the double bond of dihydro-naphthalenes $14 \mathbf{a}, \mathbf{b}$ gave the corresponding intermediates $\mathbf{1 5} \mathbf{a}-\mathbf{d}$ and subsequent formation of the benzylic carbocations 16a-d occurred, the latter of which reacted further by ring closure or $\beta$-elimination resulting in $( \pm)$-17a-d or 18a,c respectively, depending on the substitution pattern of the aryl moieties. The formation of the carba analogue of $( \pm)$-13a $\left(\mathrm{O}-5=\mathrm{CH}_{2}\right)$ or 12a could not be observed. These results also clearly supported the above mentioned cationic mechanism of the Heck-oxyarylation process.

\section{EXPERIMENTAL SECTION}

\section{General Procedures}

All reagents and organic compounds used were purchased from Sigma-Aldrich. ${ }^{1} \mathrm{H}$ and ${ }^{13} \mathrm{C}$ NMR spectra were recorded at $360 \mathrm{MHz}$ and $90 \mathrm{MHz}$, respectively with a Brucker AM-360 instrument in $\mathrm{CDCl}_{3}$ with TMS as internal standard. The chemicals shifts are given in $\delta$ (ppm). Precoated silica gel plates (Kieselgel 60 F 254,
$0.25 \mathrm{~mm}$ Merck) were applied for analytical and preparative TLC. The ESI-TOF MS measurements were performed on a MicroTOF-Q instrument (Bruker Daltonik $\mathrm{GmbH}$, Bremen, Germany). The yield of $( \pm)-\mathbf{8 b}-\mathbf{d}$ and $( \pm)-\mathbf{1 2 b}-\mathbf{d}$ belongs to an about $50 \%$ conversion of $( \pm)-\mathbf{4 a}$.

\section{General Procedure for the Heck-oxyarylation Reaction}

Palladium-chloride (177 mg, $1 \mathrm{mmol}$ ) and lithium chloride ( $84 \mathrm{mg}, 2 \mathrm{mmol}$ ) were magnetically stirred in dry acetone $(10 \mathrm{~mL})$ at room temperature. After $15 \mathrm{~min}$. the $2 \mathrm{H}$-chromene derivative $(1 \mathrm{mmol})$ in dry acetone $(10$ $\mathrm{mL}$ ) was added and the reaction mixture stirred again for $15 \mathrm{~min}$., followed by the addition of the 2chloromercuriphenol derivative $(1 \mathrm{mmol})$ suspended in dry acetone $(10 \mathrm{~mL})$. Stirring was continued for $3 \mathrm{~h}$. Then brine was added to the reaction mixture and it was filtered off on Celite pad to remove the $\operatorname{Pd}(0)$. The products were extracted with ethyl acetate, washed with water and dried over magnesium sulfate. The solvent was removed under reduced pressure to give a viscous oil, whose components were separated by preparative TLC using hexane: ethyl acetate 4:1 as eluent.

(土)-(6S*,6aR*,11aR*)-8,9-Methylenedioxy-6phenylpterocarpan $\mathbf{( 8 b )}$

Colorless crystals, $76 \mathrm{mg}(8 \%)$, m.p. $=192-194{ }^{\circ} \mathrm{C} ; R_{\mathrm{f}}$ $=0.8$ (hexane:ethyl acetate $=9: 1) ;{ }^{1} \mathrm{H}$ NMR: $3.48(1 \mathrm{H}$, $\mathrm{dd}, J=6.8 \mathrm{~Hz}, J=10.4 \mathrm{~Hz}, \mathrm{H}-6 \mathrm{a}), 4.46(1 \mathrm{H}, \mathrm{d}, J=10.8$ Hz, H-6), 5.59 (1H, d, $J=6.4$ Hz, H-11a), 5.73 (1H, s, $\mathrm{H}-10), 5.85\left(2 \mathrm{H}, 2 \mathrm{~s}, \mathrm{OCH}_{2} \mathrm{O}\right), 6.47(1 \mathrm{H}, \mathrm{s}, \mathrm{H}-7), 7.02$ $(1 \mathrm{H}, \mathrm{d}, J=8.2 \mathrm{~Hz}, \mathrm{H}-4), 7.09$ (1H, t $J=7.3 \mathrm{~Hz}, \mathrm{H}-2)$, 7.29-7.33 $\left(3 \mathrm{H}_{\text {arom }}, \mathrm{m}, \mathrm{H}-2^{\prime}, 3,6^{\prime}\right), 7.41-7.43\left(3 \mathrm{H}_{\text {arom }}, \mathrm{s}, \mathrm{H}-\right.$ $\left.3^{\prime}, 4^{\prime}, 5^{\prime}\right), 7.6(1 \mathrm{H}, \mathrm{d}, J=7.5 \mathrm{~Hz}, \mathrm{H}-1) ;{ }^{13} \mathrm{C} \mathrm{NMR}: 46.8$ (C-6a), 79,3 (C-11a), 79.6 (6), 93.4 (10), 101,1 $\left(\mathrm{OCH}_{2} \mathrm{O}\right), 106,2$ (7), 117.7 (4), 119,6, 121.8 (1), 128.1, 128.7, 128.9, 130.2 (3), 130.5 (2), 137.9, 141.0 (8), 147.9 (9), 154.1, 155.6; HRMS $(\mathrm{m} / \mathrm{z})$ calcd. for $\mathrm{C}_{22} \mathrm{H}_{16} \mathrm{NaO}_{4}$ 367.094, found [M+Na] 367.092 .

(土)-(6S*,6aR*,11aR*)-8,9-Dimethoxy-6-

phenylpterocarpan $(\mathbf{8 c})$

Colorless crystals, $57 \mathrm{mg}(13 \%)$, m.p. $=160-162{ }^{\circ} \mathrm{C} ; R_{\mathrm{f}}$ $=0.3$ (hexane:ethyl acetate $=4: 1) ;{ }^{1} \mathrm{H}$ NMR: $3.50(1 \mathrm{H}$, $\mathrm{dd}, J=6.8 \mathrm{~Hz}, J=10.8 \mathrm{~Hz}, \mathrm{H}-6 \mathrm{a}), 3.5\left(3 \mathrm{H}, \mathrm{s}, \mathrm{OCH}_{3}\right)$, $3.84\left(3 \mathrm{H}, \mathrm{s}, \mathrm{OCH}_{3}\right), 4.44(1 \mathrm{H}, \mathrm{d}, J=10.8 \mathrm{~Hz}, \mathrm{H}-6), 5.60$ $(1 \mathrm{H}, \mathrm{d}, J=6.8 \mathrm{~Hz}, \mathrm{H}-11 \mathrm{a}), 5.78(1 \mathrm{H}, \mathrm{s}, \mathrm{H}-10), 6.54$ (1H, s, H-7), $7.04(1 \mathrm{H}, \mathrm{d}, J=8.2 \mathrm{~Hz}, \mathrm{H}-4), 7.09(1 \mathrm{H}, \mathrm{t}$, $J=7.5 \mathrm{~Hz}, \mathrm{H}-2), 7.30-7.33\left(3 \mathrm{H}_{\text {arom }}, \mathrm{m}, \mathrm{H}-2^{\prime}, 3,6^{\prime}\right), 7.40-$ $7.42\left(3 \mathrm{H}_{\text {arom }}, \mathrm{s}, \mathrm{H}-3^{\prime}, 4^{\prime}, 5^{\prime}\right), 7.63(1 \mathrm{H}, \mathrm{dd}, J=7.5 \mathrm{~Hz}, J=$ $1.4 \mathrm{~Hz}, \mathrm{H}-1) ;{ }^{13} \mathrm{C}$ NMR : 47.4 (C-6a), $55.9\left(\mathrm{CH}_{3}\right), 56.2$ $\left(\mathrm{CH}_{3}\right), 79.2$ (C-11a), 79.5 (6), 94.9 (10), 110.1 (7), 116.3, 117.6 (4), 119.7, 121.8 (1), 128.1, 128.6, 130.0 (3), 130.7 (2), 137.9, 142.9 (8), 150.0 (9), 153.5, 155.3; HRMS $(m / z)$ calcd. for $\mathrm{C}_{23} \mathrm{H}_{20} \mathrm{NaO}_{4} 383.125$, found $[\mathrm{M}+\mathrm{Na}]^{+} 383.124$. 
( \pm )-(6S*,6aR*,11aR*)-8,9-Diethoxy-6-

phenylpterocarpan $(\mathbf{8 d})$

Colorless crystals, $75 \mathrm{~g}(20 \%)$, m.p. $=153.5-154.8^{\circ} \mathrm{C}$; $R_{\mathrm{f}}=0.5$ (hexane:ethyl acetate $=9: 1$ ); ${ }^{1} \mathrm{H}$ NMR: 1.25 $\left(3 \mathrm{H}, \mathrm{t}, J=6.8 \mathrm{~Hz}, \mathrm{CH}_{3}\right), 1.43\left(3 \mathrm{H}, \mathrm{t}, J=6.8 \mathrm{~Hz}, \mathrm{CH}_{3}\right)$, $3.48(1 \mathrm{H}, \mathrm{dd}, J=6.8 \mathrm{~Hz}, J=11.1 \mathrm{~Hz}, \mathrm{H}-6 \mathrm{a}), 3.71(2 \mathrm{H}$, $\left.\mathrm{m}, \mathrm{OCH}_{2}\right), 4.03\left(2 \mathrm{H}, \mathrm{m}, \mathrm{OCH}_{2}\right), 4.44(1 \mathrm{H}, \mathrm{d}, J=11.1$ $\mathrm{Hz}, \mathrm{H}-6), 5.58(1 \mathrm{H}, \mathrm{d}, J=6.8 \mathrm{~Hz}, \mathrm{H}-11 \mathrm{a}), 5.82(1 \mathrm{H}, \mathrm{s}$, H-10), 6.52 (1H, s, H-7), 7.03 (1H, d, J=7.9 Hz, H-4), $7.10(1 \mathrm{H}, \mathrm{t}, J=7.5 \mathrm{~Hz}, \mathrm{H}-2), 7.29-7.33\left(3 \mathrm{H}_{\text {arom }}, \mathrm{m}, \mathrm{H}-\right.$ $\left.2^{\prime}, 3,6^{\prime}\right), 7.39-7.41\left(3 \mathrm{H}_{\text {arom }}, \mathrm{s}, \mathrm{H}-3^{\prime}, 4^{\prime}, 5^{\prime}\right), 7.62(1 \mathrm{H}, \mathrm{d}, J=$ $7.5 \mathrm{~Hz}, \mathrm{H}-1) ;{ }^{13} \mathrm{C} \mathrm{NMR}: 14.7\left(\mathrm{CH}_{3}\right), 47.4$ (C-6a), 64.9 $\left(\mathrm{CH}_{2}\right), 79.2$ (C-11a), 79.5 (6), 94.9 (10), 110.1 (7), 116.3, 117.6 (4), 119.7, 121.8 (1), 128.1, 128.6, 130.0 (3), 130.7 (2), 137.9, 142.9 (8), 150.0 (9), 153.5, 155.3; HRMS $(m / z)$ calcd. for $\mathrm{C}_{25} \mathrm{H}_{24} \mathrm{NaO}_{4} 411.157$, found $[\mathrm{M}+\mathrm{Na}]^{+}$411.156.

( \pm )-(6R*,12R*)-6,11-Methano-2,3-methylenedioxy-6phenyl-6H,11H-dibenzo[d,g][1,3]dioxocine (12b) Colorless crystals, $21 \mathrm{mg}(2 \%)$, m.p. $=196-198^{\circ} \mathrm{C} ; R_{\mathrm{f}}$ $=0.7$ (hexane:ethyl acetate $=9: 1) ;{ }^{1} \mathrm{H}$ NMR: $2.58(2 \mathrm{H}$, $\mathrm{d}, J=2.8 \mathrm{~Hz}, \mathrm{H}-13), 3.95(1 \mathrm{H}, \mathrm{s}, \mathrm{H}-12), 5.85$ (2H, 2s, $\left.\mathrm{OCH}_{2} \mathrm{O}\right), 6.56(1 \mathrm{H}, \mathrm{s}, \mathrm{H}-4), 6.69(1 \mathrm{H}, \mathrm{s}, \mathrm{H}-1), 6.91(1 \mathrm{H}$, t, $J=7.2 \mathrm{~Hz}, \mathrm{H}-10), 7.02(1 \mathrm{H}, \mathrm{d}, J=7.9 \mathrm{~Hz}, \mathrm{H}-8), 7.14$ $(1 \mathrm{H}, \mathrm{t}, J=7.7 \mathrm{~Hz}, \mathrm{H}-9), 7.21(1 \mathrm{H}, \mathrm{d}, J=7.2 \mathrm{~Hz}, \mathrm{H}-11)$, 7.40-7.47 $\left(3 \mathrm{H}_{\text {arom }}, \mathrm{m}, \mathrm{H}-3^{\prime}, 4^{\prime}, 5^{\prime}\right), 7.72(2 \mathrm{H}, \mathrm{d}, J=6.8$ $\left.\mathrm{Hz}, \mathrm{H}-2^{\prime}, 6^{\prime}\right) ;{ }^{13} \mathrm{C} \mathrm{NMR}: 33.5$ (13), 33.8 (12), 98.5 (6), 101.1 (4), $103.1\left(\mathrm{OCH}_{2} \mathrm{O}\right), 110.2(1), 116.6(8), 116.7$, 121.3 (11), 126.7, 126.8, 126.9, 127.9 (9), 128.3, 128.7 (10), 141.4, 143.7 (2), 145.6 (3), 148.8, 151.9; HRMS $(\mathrm{m} / \mathrm{z})$ calcd. for $\mathrm{C}_{22} \mathrm{H}_{16} \mathrm{NaO}_{4} 367.094$, found $[\mathrm{M}+\mathrm{Na}]^{+}$ 367.092 .

( \pm )-(6R*,12R*)-2,3-Dimethoxy-6,11-methano-6-phenyl6H,11H-dibenzo $[d, g][1,3]$ dioxocine $(\mathbf{1 2 c})$

Colorless crystals, $23 \mathrm{mg}(5 \%)$, m.p. $=167-169^{\circ} \mathrm{C} ; R_{\mathrm{f}}$ $=0.2$ (hexane:ethyl acetate $=4: 1) ;{ }^{1} \mathrm{H}$ NMR: $2.34(1 \mathrm{H}$, $\left.\mathrm{dd}, J_{\mathrm{Hax}}, \mathrm{H}-12=3.2 \mathrm{~Hz}, J_{\text {Hax }, \text { Heq }}=13.3 \mathrm{~Hz}, \mathrm{H}-13_{\mathrm{ax}}\right), 2.41$ $\left(1 \mathrm{H}, \mathrm{dd}, J_{\mathrm{Heq}}, \mathrm{H}-12=2.8 \mathrm{~Hz}, J_{\mathrm{Heq}, \mathrm{Hax}}=13.3 \mathrm{~Hz}, \mathrm{H}-13_{\mathrm{eq}}\right)$, 3.81 and $3.85\left(6 \mathrm{H}, 2 \mathrm{~s}, 2 \mathrm{OCH}_{3}\right), 3.98(1 \mathrm{H}, \mathrm{t}, J=2.8 \mathrm{~Hz}$, $\mathrm{H}-12), 6.61(1 \mathrm{H}, \mathrm{s}, \mathrm{H}-4), 6.72(1 \mathrm{H}, \mathrm{s}, \mathrm{H}-1), 6.90(1 \mathrm{H}, \mathrm{t}$, $J=7.5 \mathrm{~Hz}, \mathrm{H}-10), 6.94(1 \mathrm{H}, \mathrm{d}, J=7.5 \mathrm{~Hz}, \mathrm{H}-8), 7.15$ $(1 \mathrm{H}, \mathrm{t}, J=8.6 \mathrm{~Hz}, \mathrm{H}-9), 7.24(1 \mathrm{H}, \mathrm{d}, J=7.5 \mathrm{~Hz}, \mathrm{H}-11)$, 7.41-7.46 $\left(3 \mathrm{H}_{\text {arom }}, \mathrm{m}, \mathrm{H} 3^{\prime}, 4^{\prime}, 5^{\prime}\right), 7.76(2 \mathrm{H}, \mathrm{d}, J=8.2$ $\left.\mathrm{Hz}, \mathrm{H}-2^{\prime}, 6^{\prime}\right)$; ${ }^{13} \mathrm{C}$ NMR: 33.5 (13), 33.8 (12), $55.9\left(\mathrm{CH}_{3}\right)$, $56.6\left(\mathrm{CH}_{3}\right), 98.5$ (6), 101.1 (4), 110.2 (1), 116.6 (8), 116.7, 121.3 (11), 126.7, 126.8, 126.9, 127.9 (9), 128.3, 128.7 (10), 141.4, 143.7 (2), 145.6 (3), 148.8, 151.9; HRMS $(m / z)$ calcd. for $\mathrm{C}_{23} \mathrm{H}_{20} \mathrm{NaO}_{4} 383.125$, found $[\mathrm{M}+\mathrm{Na}]^{+} 383.124$.

( \pm )-(6R*,12R*)-2,3-Diethoxy-6,11-methano-6-phenyl$6 H, 11 H$-dibenzo $[d, g][1,3]$ dioxocine $(\mathbf{1 2 d})$

Colorless crystals, $85 \mathrm{mg}(23 \%)$, m.p. $=115-118{ }^{\circ} \mathrm{C}$; $R_{\mathrm{f}}=0.4$ (hexane:ethyl acetate $=9: 1$ ); ${ }^{1} \mathrm{H}$ NMR: 1.40 $\left(6 \mathrm{H}, \mathrm{t}, J=6.8 \mathrm{~Hz}, 2 \mathrm{CH}_{3}\right), 2.32\left(1 \mathrm{H}, \mathrm{dd}, J_{\mathrm{Heq}}, \mathrm{H}-12=\right.$ $\left.2.8 \mathrm{~Hz}, J_{\text {Heq, Hax }}=13.3 \mathrm{~Hz}, \mathrm{H}-13_{\text {eq }}\right), 2.40(1 \mathrm{H}, \mathrm{dd}$, $\left.J_{\text {Hax }}, \mathrm{H}-12=3.2 \mathrm{~Hz}, J_{\text {Hax,Heq }}=13.3 \mathrm{~Hz}, \mathrm{H}-13_{\mathrm{ax}}\right), 3.96$ $(1 \mathrm{H}, \mathrm{t}, J=2.8 \mathrm{~Hz}, \mathrm{H}-12), 4.02\left(4 \mathrm{H}, \mathrm{m}, 2 \mathrm{OCH}_{2}\right), 6.60$ $(1 \mathrm{H}, \mathrm{s}, \mathrm{H}-4), 6.76(1 \mathrm{H}, \mathrm{s}, \mathrm{H}-1), 6.91(1 \mathrm{H}, \mathrm{t}, J=7.2 \mathrm{~Hz}$, $\mathrm{H}-10), 7.02(1 \mathrm{H}, \mathrm{d}, J=7.9 \mathrm{~Hz}, \mathrm{H}-8), 7.14(1 \mathrm{H}, \mathrm{t}, J=$ $7.9 \mathrm{~Hz}, \mathrm{H}-9), 7.23$ (1H, d, $J=7.5 \mathrm{~Hz}, \mathrm{H}-11), 7.40-7.47$ $\left(3 \mathrm{H}_{\text {arom }}, \mathrm{m}, \mathrm{H} 3^{\prime}, 4^{\prime}, 5^{\prime}\right), 7.75(2 \mathrm{H}, \mathrm{d}, J=7.9 \mathrm{~Hz}, \mathrm{H}-$ $\left.2^{\prime}, 6^{\prime}\right) ;{ }^{13} \mathrm{C}$ NMR: $14.7\left(\mathrm{CH}_{3}\right), 33.5$ (13), 33.8 (12), 64.9 $\left(\mathrm{CH}_{2}\right), 98.5$ (6), 101.1 (4), 110.2 (1), 116.6 (8), 116.7, 121.3 (11), 126.7, 126.8, 126.9, 127.9 (9), 128.3, 128.7 (10), 141.4, 143.7 (2), 145.6 (3), 148.8, 151.9; HRMS $(\mathrm{m} / z)$ calcd. for $\mathrm{C}_{25} \mathrm{H}_{24} \mathrm{NaO}_{4} 411.157$, found $[\mathrm{M}+\mathrm{Na}]^{+}$ 411.156 .

(土)-(6aR*,11aS*)-5,6,6a,11a-

Tetrahydrobenzo[d]naphtho[1,2-b]furan (17a)

White crystals, $59 \mathrm{mg}(14 \%)$, m.p. $=39-40{ }^{\circ} \mathrm{C}$ (Ref. 18, $40{ }^{\circ} \mathrm{C}$ ); $R_{\mathrm{f}}=0.7$ (hexane: ethyl acetate $=9: 1$ ); ${ }^{1} \mathrm{H}$ NMR: 1.86 (2H, m, H-6), 2.59 (2H, m, H-5), 3,60 (1H, m, H6a), $5.58(1 \mathrm{H}, \mathrm{d}, J=8.4 \mathrm{~Hz}, \mathrm{H}-11 \mathrm{a}), 6.70(1 \mathrm{H}, \mathrm{d}, J=$ $8 \mathrm{~Hz}, \mathrm{H}-10), 6.81$ (1H, dt, $J=7.4 \mathrm{~Hz}, J=1.48 \mathrm{~Hz}, \mathrm{H}-8)$, $7.05\left(2 \mathrm{H}_{\text {arom }}, \mathrm{m}, \mathrm{H}-2,4\right), 7.18\left(3 \mathrm{H}_{\text {arom }}, \mathrm{m}, \mathrm{H}-3,7,9\right)$, $7.46(1 \mathrm{H}, \mathrm{dd}, J=7.2 \mathrm{~Hz}, J=1.6 \mathrm{~Hz}, \mathrm{H}-1)$; HRMS $(m / z)$ calcd. for $\mathrm{C}_{16} \mathrm{H}_{14} \mathrm{NaO}$ 245.094, found: $[\mathrm{M}+\mathrm{Na}]^{+}$ 245.090.

(士)-(6aR*,11aS*)-8,9-Methylenedioxy-5,6,6a,11atetrahydrobenzo[d]naphtho[1,2-b]furan (17b)

White crystals, $152 \mathrm{mg}(29 \%)$, m.p. $=84-86{ }^{\circ} \mathrm{C} ; R_{\mathrm{f}}=$ 0.6 (hexane: ethyl acetate $=9: 1)$; ${ }^{1} \mathrm{H}$ NMR: $1.87(2 \mathrm{H}, \mathrm{m}$, H-6), 2.67 (2H, m, H-5), 3.57 (1H, m, H-6a), $5.65(1 \mathrm{H}$, $\mathrm{d}, J=8.6 \mathrm{~Hz}, \mathrm{H}-11 \mathrm{a}), 5.88\left(2 \mathrm{H}, \mathrm{d}, J=5.4 \mathrm{~Hz}, \mathrm{OCH}_{2} \mathrm{O}\right)$, $6.37(1 \mathrm{H}, \mathrm{s}, \mathrm{H}-10), 6.70(1 \mathrm{H}, \mathrm{s}, \mathrm{H}-7), 7.14(1 \mathrm{H}, \mathrm{d}, J=$ $6.8 \mathrm{~Hz}, \mathrm{H}-4), 7.26(2 \mathrm{H}, \mathrm{m}, \mathrm{H}-2,3), 7.5(1 \mathrm{H}, \mathrm{d}, J=6.5$ $\mathrm{Hz}, \mathrm{H}-1)$; HRMS $(m / z)$ calcd. for $\mathrm{C}_{17} \mathrm{H}_{14} \mathrm{NaO}_{3} 289.084$, found: $[\mathrm{M}+\mathrm{Na}]^{+} 289.080$.

(土)-(6aR*,11aS*)-3-Methoxy-5,6,6a,11a-

tetrahydrobenzo[d] naphtho[1,2-b]furan $(\mathbf{1 7 c})$

Yellow oil, $30 \mathrm{mg}(3 \%) ; R_{\mathrm{f}}=0.6$ (hexane:ethyl acetate $=7: 3) ;{ }^{1} \mathrm{H}$ NMR: 1,93 (2H, m, H-6), 2.62 (2H, m, H-5), 3.64 (1H, m, H-6a), 3.77 (3H, d, $J=5.8 \mathrm{~Hz}, \mathrm{OMe}), 5.63$ $(1 \mathrm{H}, \mathrm{d}, J=8.6 \mathrm{~Hz}, \mathrm{H}-11 \mathrm{a}), 7.05$ (7 $\mathrm{H}_{\text {arom }}, \mathrm{m}, \mathrm{H}-1,2,4,7$, 8, 9, 10); HRMS $(m / z)$ calcd. for $\mathrm{C}_{16} \mathrm{H}_{14} \mathrm{NaO} 275.104$, found: $[\mathrm{M}+\mathrm{Na}]^{+} 275.100$.

(土)-(6aR*,11aS*)-3-Methoxy-8,9-methylenedioxy5,6,6a,11a-tetrahydrobenzo[d]naphtho[1,2-b]furan (17d)

Yellow oil, $311 \mathrm{mg}(30 \%) ; R_{\mathrm{f}}=0.6$ (hexane:ethyl acetate $=7: 3)$; ${ }^{1} \mathrm{H}$ NMR: $1.86(2 \mathrm{H}, \mathrm{m}, \mathrm{H}-6), 2.66(2 \mathrm{H}, \mathrm{m}$, H-5), 3.55 (1H, m, H-6a), 3.80 (3H, s, OMe), $5.63(1 \mathrm{H}$, $\mathrm{d}, J=8.3 \mathrm{~Hz}, \mathrm{H}-11 \mathrm{a}), 5.88\left(2 \mathrm{H}, \mathrm{d}, J=4.7 \mathrm{~Hz}, \mathrm{OCH}_{2} \mathrm{O}\right)$, $6.36(1 \mathrm{H}, \mathrm{s}, \mathrm{H}-10), 6.69(1 \mathrm{H}, \mathrm{d}, J=2.1 \mathrm{~Hz}, \mathrm{H}-4), 6.70$ (1H, s, H-7), 6.84 (1H, dd, $J=8.3 \mathrm{~Hz}, J=2.2 \mathrm{~Hz}, \mathrm{H}-2)$, $7.42(1 \mathrm{H}, \mathrm{d}, J=8.3 \mathrm{~Hz}, \mathrm{H}-1)$; HRMS $(\mathrm{m} / \mathrm{z})$ calcd. for $\mathrm{C}_{18} \mathrm{H}_{16} \mathrm{NaO}_{4}$ 319.094, found: [M+Na] 319.093 . 
2-(3,4-Dihydronaphthalen-2-yl)phenol (18a)

Brown oil, $32 \mathrm{mg}(3 \%) ; R_{\mathrm{f}}=0.3$ (hexane:ethyl acetate = 9:1); ${ }^{1} \mathrm{H}$ NMR: 2.69 (2H, t $\left.J=8.2 \mathrm{~Hz}, \mathrm{H}-4\right), 2.99$ $(2 \mathrm{H}, \mathrm{t}, J=8 \mathrm{~Hz}, \mathrm{H}-3), 5.56(1 \mathrm{H}, \mathrm{s}, \mathrm{OH}), 6.73(1 \mathrm{H}, \mathrm{s}, \mathrm{H}-$ 1), $6.96(2 \mathrm{H}, \mathrm{d}, J=7.6 \mathrm{~Hz}, \mathrm{H}-5, \mathrm{H}-8), 7.09-7.24$ $\left(6 \mathrm{H}_{\text {arom }}, \mathrm{m}, \mathrm{H}-6,7, \mathrm{H}_{\text {phenol- }}-3,4,5,6\right)$; HRMS $(\mathrm{m} / \mathrm{z})$ calcd. for $\mathrm{C}_{16} \mathrm{H}_{14} \mathrm{NaO}$ 245.094, found: $[\mathrm{M}+\mathrm{Na}]^{+} 245.091$.

2-(6-Methoxy-3,4-dihydronaphthalen-2-yl)phenol (18c) Brown oil, $361 \mathrm{mg}(48 \%) ; R_{\mathrm{f}}=0.3$ (hexane:ethyl acetate $=7: 3) ;{ }^{1} \mathrm{H}$ NMR: $2.64(2 \mathrm{H}, \mathrm{t}, J=7.9 \mathrm{~Hz}, \mathrm{H}-4), 2.93$ $(2 \mathrm{H}, \mathrm{t}, J=7.9, \mathrm{H}-3), 3.81(3 \mathrm{H}, \mathrm{s}, \mathrm{OMe}), 5.63(1 \mathrm{H}, \mathrm{s}$, $\mathrm{OH}), 6.66$ (1H, s, H-1), 6.71-7.19 (7 $\mathrm{H}_{\text {arom }}, \mathrm{m}, \mathrm{H}-5,7,8$, $\mathrm{H}_{\text {phenol- }} 3$, 4, 5, 6); HRMS $(\mathrm{m} / \mathrm{z})$ calcd. for $\mathrm{C}_{17} \mathrm{H}_{16} \mathrm{NaO}_{2}$ 275.104, found: $[\mathrm{M}+\mathrm{Na}]^{+} 275.102$.

Acknowledgements. We are grateful to the National Research Foundations (OTKA K81701 and TAMOP 4.2.2./B-10/1) for the financial support of our research.

\section{REFERENCES}

1. P. M. Dewick and J. B. Harborne, Flavonoids, Advences in Research Since 1986, Chapman and Hall: London (1994) p 166.

2. D. R. Perrin and A. M. Cruickshank, Phytochemistry 8 (1969) 971-978.

3. T. A. Engler, O. K. Lynch, J. P. Reddy, and E. S. Gregory, Bioorg. Med. Chem. Lett. 3 (1993) 1229-1232.

4. M. Nakagawa, K. Nakanishi, L. L. Darko, and J. A. Vick, Tetrahedron Lett. 23 (1982) 3855-3858.

5. H. Suginome and T. Iwadare, Bull. Chem. Soc. Jpn. 39 (1986) 1535-1541.

6. L. Farkas, Á. Gottsegen, M. Nógrádi, and S. Antus, J. Chem. Soc., Perkin Trans. 11974 305-312.
7. H. Horino and N. Inoue, J. Chem. Soc. Chem. Commun. (1976) 500-501.

8. Y. Ozaki, K. Mochida, and S.-W. Kim, J. Chem. Soc. Chem. Commun. (1988) 374-375.

9. A. Gopalsamy and K. K. Balasubramanian, J. Chem. Soc., Chem. Commun. (1988) 28-29.

10. T. A. Engler, J. P. Reddy, K. D. Combrink, and D. J. VanderVelde, J. Org. Chem. 55 (1990) 1248-1254.

11. T. G. van Aardt, P. S. van Heerden, and D. Ferreira, Tetrahedron Lett. 39 (1998) 3881-3884.

12. L. J. Gonzalez, M. A. Corral, M. M. Dorado, and I. R. Garcia, Chem. Commun. (2005) 2689-2691.

13. L. J. Gonzalez, S. G. Munoz, M. A. Corral, M. M. Dorado, and I. R. Garcia, Chem. Eur. J. 12 (2006) 8762-8769.

14. R. Skouta and Ch. J. Li, Terahedron Lett. 48 (2007) 8343-8346.

15. R. S. Khupse and P. W. Erhardt, Organic Letters 10 (2008) 5007-5010.

16. M. A. Calter and N. Li, Organic Letters 13 (2011) 3686-3689.

17. J. C. Breytenbach and G. J. H. Rall, J. Chem. Soc. Perkin Trans. 1. 1980 1804-1809.

18. M. Ishiguro, T. Tatsuoka, and N. Nakatsuka, Tetrahedron Lett. 23 (1982) 3859-3862.

19. D. D. Narkhede, P. R. Iyer, and C. S. R. Iyer, Tetrahedron 46 (1990) 2031-2034.

20. A. L. Coelho, M. L. A. A. Vasconcellos, A. B. C. Simas, J. A. Rabi, and P. R. R. Costa, Synthesis (1992) 914-916.

21. R. A. Lichtenfels, A. L. Coelho, and P. R. R. Costa, J. Chem. Soc. Perkin Trans. 11995 949-951.

22. A. J. M. Da Silva, A. L. Coelho, A. B. C. Simas, R. A. M. Moraes, D. A. Pinheiro, F. F. A. Fernandes, E. Z. Aruda, P. R. R. Costa, and P. A. Melo, Bioorg. Med. Chem. Lett. 14 (2004) 431-435.

23. A. L. Tőkés, Gy. Litkei, K. Gulácsi, S. Antus, E. Baitz-Gács, Cs. Szántay, and L. L. Darkó, Tetrahedron 55 (1999) 9283-9296.

24. T. Patonay, D. Molnár, and Z. Murányi, Bull. Soc. Chim. Fr. 132 (1995) 233-242. 